\title{
Emotion processing in Joint Hypermobility: a potential link to the neural bases of anxiety and related somatic symptoms in collagen anomalies.
}

\section{Running title: Emotional processing in the Joint Hypermobility Syndrome}

Mallorquí-Bagué N, MSc ${ }^{1,2,3}$ Bulbena A, MD, MSc(Cantab) ${ }^{1,4,5}$, Roé-Vellvé N. $\mathrm{PhD}^{3}$,

Hoekzema E, $\mathrm{PhD}^{3,6}$, Carmona S, $\mathrm{PhD}^{3,7}$, Barba-Müller E, $\mathrm{MSc}^{1,3}$, Fauquet J, $\mathrm{PhD}^{3,8}$, Pailhez G, MD, $\mathrm{PhD}^{1,4,5}$,Vilarroya O,MD, $\mathrm{PhD}^{1,3}$

${ }^{1}$ Doctorate program in Psychiatry. Department of Psychiatry and Forensic Medicine. School of Medicine. Campus de la Universitat Autonoma de Barcelona (UAB). Spain.

${ }^{2}$ Psychiatry, Psychology and Psychosomatics department. Institut Universitari Quirón Dexeus. Barcelona. Spain.

${ }^{3}$ Neuroimaging Research Group. Fundació IMIM. Doctor Aiguader, 88. 08003 Barcelona, Spain.

${ }^{4}$ Fundació IMIM (Hospital del Mar Medical Research Institute), Barcelona. Spain.

${ }^{5}$ Anxiety Unit.Institute of Neuropsychiatry and Addictions (INAD).Hospital del Mar. Universitat Autònoma de Barcelona. Barcelona. Spain.

${ }^{6}$ Netherlands Institute for Neuroscience, Meibergdreef 47, 1105 BA Amsterdam, the Netherlands

${ }^{7}$ Centro de Investigación Biomédica en Red de Salud mental, Spain (SC, MD); Instituto de Investigación Sanitaria Gregorio Marañón, Madrid, Spain; Departamento de Bioingeniería e Ingeniería Aeroespacial, Universidad Carlos III de Madrid, Madrid, Spain

${ }^{8}$ Dept. Psicobiologia i Metodologia de les Ciències de la Salut. Campus de la UAB. 08193 Bellaterra (Cerdanyola del Vallés), Barcelona, Spain

Words of the manuscript: 3018.-Words of the Abstract: 270.-Number of tables: 2.Number of Figures: 1. - Supplemental information: 0.

Correspondence concerning this article should be addressed to Núria Mallorquí Bagué Passeig Marítim 25, 08003. Barcelona. Spain. E-mail: nmallorqui@live.com. Telf: (+34) 932483495/ Fax: (+34) 932483445. 


\section{Abstract}

Background: Joint Hypermobility Syndrome (JHS) has repeatedly been associated with anxiety and anxiety disorders, fibromyalgia, irritable bowel syndrome and temporomandibular joint disorder. However the neural underpinnings of these associations still remain unclear. This study explored brain responses to facial visual stimuli with emotional cues using fMRI techniques in general population with different ranges of hypermobility. Methods: 51 non-clinical volunteers (33 women) completed state and trait anxiety questionnaire measures, were assessed with a clinical examination for hypermobility (Beighton system) and performed an emotional face processing paradigm during functional neuroimaging. Results: Trait anxiety scores did significantly correlate with both state anxiety and hypermobility scores. BOLD signals of the hippocampus did positively correlate with hypermobility scores for the crying faces versus neutral faces contrast in ROI analyses. No results were found for any of the other studied ROIs. Additionally, hypermobility scores were also associated with other key affective processing areas (i.e. the middle and anterior cingulate gyrus, fusiform gyrus, parahippocampal region, orbitofrontal cortex and cerebellum) in the whole brain analysis.

Conclusions: Hypermobility scores are associated with trait anxiety and higher brain responses to emotional faces in emotion processing brain areas (including hippocampus) described to be linked to anxiety and somatic symptoms. These findings increase our understanding of emotion processing in people bearing this heritable variant of collagen and the mechanisms through which vulnerability to anxiety and somatic symptoms arises in this population.

Key words: Functional magnetic resonance imaging (fMRI), Emotion, Joint hypermobility, Anxiety, Psychosomatics 


\section{Introduction}

Joint hypermobility syndrome (JHS) is an inherit connective tissue condition that represents a qualitative variation in the structural protein collagen. The estimated prevalence of JHS in the general (Western European) population ranges between 10-15\% $[1,2]$ and it is more frequent in women (3:1). Although JHS is a common and disturbing disorder, it remains poorly recognised.

Our research group found an overrepresentation of JHS among people with anxiety but especially among the so called endogenous anxiety disorders (i.e.: panic, agoraphobic and social phobia) [3, 4]. Also, individuals with JHS often present stress-sensitive illnesses such as irritable bowel syndrome, fibromyalgia, temporomandibular joint disorder and chronic fatigue syndrome [5] as well as autonomic abnormalities [6]. Likewise, JHS is also overrepresented among the general population with a high range of trait anxiety [7] and it has recently been described as a risk factor for developing anxiety disorders in a longitudinal study [8].

The mechanisms underlying the association between JHS and anxiety-related disorders are still unclear. However, a structural neuroimaging study of healthy non-anxious individuals have shown that people with features of hypermobility manifest larger amygdala volume bilaterally compared to participants without any hypermobility [9]. Furthermore, recent findings revealed interoceptive sensitivity mediated the relationship between state anxiety and hypermobility in a non-clinical sample. Also in this last study the hypermobile participants, when compared to the non-hypermobile, displayed heightened neural reactivity to sad and angry scenes within brain regions implicated in anxious feeling states; notably insular cortex [10] 
The aim of the present study was to characterize how hypermobility scores are associated with brain activity in response to facial stimuli with emotional cues. Facial expressions are a classical way to probe automatic emotional responses [11]. We hypothesize that hypermobility measures will be positively correlated with higher anxiety measures and with a higher BOLD signal in key affective processing regions that could underlie anxiety and somatic symptoms (i.e.: amygdala, hippocampus, insular cortex, and anterior cingulate cortex) [12-16] in response to stimuli with higher emotional contents.

\section{Method}

Participants-Sixty-eight right handed non-clinical volunteers (thirty-nine women) were recruited for the study. All participants were evaluated through a medical and psychiatric history as well as assessed by two structured clinical diagnostic interviews [17][18]. None of the participants had taken psychotropic medication in the previous 12 months or had any pathology that could interfere in the hypermobility assessment. Four participants were excluded for neurological or psychiatric reasons. Thirteen more subjects were omitted from the analysis due to movement artefacts in the MR images. The final sample consisted of fifty-one participants free from any Axis I disorder, psychotropic medication or any other pathology that could interfere in the fMRI paradigm brain response and/or in the hypermobility assessment. All volunteers were given a complete description of the fMRI and clinical examination before written informed consent was obtained, and all of them voluntarily agreed to take part in the study. Parc de Salut Mar Barcelona clinical research ethical committee approval was obtained.

Measures-Participants were screened and evaluated through a medical and psychiatric history as well as assessed by the MINI structured diagnostic interview [17] and the Structured clinical interview for Axis I (SCID-I) [18]. The fifty-one non-clinical 
participants of the final sample completed the Spielberg state-trait anxiety inventory (STAI) [19], conducted a functional imaging task and were assessed with the Beighton exploration for hypermobility [20].The Beighton clinical exploration of hypermobility requires a physical examination that was conducted by a trained and experienced clinician (according to the basis of the clinical rheumatologists' standards, kappa inter-examiners reliability ranged from 0,8 to 1 ). The Beighton scoring system consists of five items (describing nine movements), that explores the joint mobility range of 5 body areas: wrists/thumb, knees, spine/hips, paired elbows and fifth metacarpo-phalangeals. The highest score is nine and an accepted cut-off point is 4/5 (man/women).

Functional imaging task- Participants were trained on the emotional face task before the fMRI session. During the acquisition the volunteers participated in an event-related paradigm of emotional facial stimuli in which two groups of images were presented: 14 images of crying faces and 14 images of neutral faces. Images were derived from the Gur et al. series and supplemented with similar valence-matched images [21]. The stimuli were displayed for $1500 \mathrm{~ms}$, followed by an interstimulus interval of between 750 to $1300 \mathrm{~ms}$, with mean trial duration of 2500 ms. Pictures presentation was completely randomized for each participant. Participants performed an incidental task: they were instructed to press a button with their right hand when an adult face was presented and with their left hand when they saw an infant.

Image acquisition parameters- Images were acquired in a Philips Achieva 3T scanner. T1weighted images were obtained using a FSPGR sequence (TR: $8.2 \mathrm{~ms}$, TE: 3.7ms, FA: $8^{\circ}$, matrix size: 256x256x180, voxel size: $0.94 \mathrm{~mm}$ x $0.94 \mathrm{~mm}$ x $1.00 \mathrm{~mm}$, gap: $0 \mathrm{~mm}$ ). An EPI-T2* sequence allowed obtaining the functional volumes, each comprising 30 three mm-thick slices(TR 3000 ms, TE: 35 ms, FA: 90º, in-plane voxel size 1.80 mm x 1.80 mm, Slice thickness $3.0 \mathrm{~mm}$, gap $=1.0 \mathrm{~mm}$, matrix size: $128 \times 128$, 30 slices). 
Image processing-Images were pre-processed within SPM8 (http://www.fil.ion.ucl.ac.uk/spm/) implemented in MATLAB 7.14 (Mathworks Inc. Sherborn, M.A.). The first three volumes of each session were discarded to remove non steady-state effects. Head motion correction was carried out by means of spatial interpolation, employing parameters derived from a six-parameter rigid body transformation with a least squares algorithm. Functional images were realigned to the first volume, normalized to the MNI EPI template, and smoothed with a12 mm FWHM kernel using SPM8.

Statistical analysis- At the first level of analysis, voxel-wise changes in Blood-oxygenlevel dependence (BOLD) response across conditions were assessed for each subject and analysed according to the general linear model. The regressors of interest were convolved with the canonical hemodynamic response function implemented in SPM and optimal parameter estimates were computed using a least squares function. A linear contrast crying face $>$ neutral face (corresponding to an increased BOLD response for the crying faces as compared to neutral faces) was applied to estimate the effect sizes for each participant and generate the associated statistical parametric map. Second level analyses were implemented for the main studied variable (i.e.: hypermobility scores) and the established crying face $>$ neutral face contrast. We checked for the dependence of the contrast values on the hypermobility scale values. This was done using SPM, by introducing the hypermobility scale as a covariate in a one-sample t-test of the entire sample. We then evaluated the effect of this covariate by converting the beta coefficient to a t-score (p-value threshold was set at 0.05, FWE-corrected). We studied hypermobility as a continuous variable because we wanted to explore the whole spectrum of joint mobility and not only the ones that accomplish the pathological threshold. The analysis was performed within a set of preselected regions of interest (ROIs) that have been described as important affective 
processing regions: amygdala, hippocampus, insular cortex, and anterior cingulate cortex [12-14]. ROI masks were obtained from the WFU pickatlas Talairach [22, 23] brain maps and $p$-value threshold was set at 0.05 , FWE-corrected. This analysis was carried out with SPM at the voxel level, by analysing only the voxels within each ROI, applying the mentioned one-sample t-test. For the areas showing a significant activation linked to hypermobility, a subsequent analysis was carried out to better illustrate the observed correlation. These areas were used as ROIs, and the mean contrast value within the ROI was plotted, for each participant, against his hypermobility score. The corresponding Pearson's R was calculated using STATA v13.0. Finally, whole brain analysis ( $p$-value threshold set at 0.001 , uncorrected) was also performed at the established crying face > neutral face contrast for exploratory purposes.

\section{Results}

The mean age of the sample was 33.31 years $(S D=4.93)$ and $64.7 \%$ were women. Sample homogeneity was granted by no significant difference of age or sex. Eleven participants (21.6\% of the sample) meet the Beighton criteria for JHS. This percentage is higher than the one described in general population in epidemiological studies, however this could be explained by the higher percentage of women in the sample (hypermobility is more frequent among women (3:1)). The range, mean and SD of the hypermobility and anxiety scores are detailed in table 1.

\section{Anxiety scores and hypermobility criteria}

The self-reported measures of anxiety-state-trait anxiety-were significantly intercorrelated across the 51 participants. Besides hypermobility scores were significantly correlated with trait anxiety scores (see Table 1). 
ROI analyses

Hypermobility was positively correlated with BOLD signal in the left hippocampus in response to the crying versus neutral face contrast $(t=4.08$; cluster $p$ (FWE-cor $)=0.019$; cluster size $=14)$. The correlation coefficient between contrast values in the activated area and hypermobility scores was found to be $r=0.286$ (see Figure 1). No more significant associations were found within hypermobility and the other preselected regions of interest.

\section{Whole brain analysis}

Results for the whole brain analysis are shown in Table 2.During the processing of crying face images, a discrete set of brain regions showed greater responses with increasing hypermobility scores. These included: right anterior and mid cingulum, right and left fusiform gyrus, left parahippocampus, hippocampus and right cerebellum (see Table 2). All these results were uncorrected with a p-threshold of 0.001 and a minimum cluster size of 10 .

\section{Discussion}

The aim of the present study was to identify the neural signatures of emotion processing associated with hypermobility. For this purpose we explored brain responses to facial visual stimuli with emotional cues in general population, using fMRI techniques. We focused the analysis on critical affective processing regions (i.e.: amygdala, hippocampus, insular cortex, and anterior cingulated cortex).

Our ROI analyses revealed a positive association between BOLD signals in the hippocampus and hypermobility scores in response to the crying face versus neutral face contrast. Importantly, studies have not only revealed that hippocampus has a crucial role in cognition, learning and memory, but also in the pathophysiology of anxiety disorders and 
emotional behaviour [24, 25]. Furthermore, neuroimaging studies have also revealed that hippocampus is vulnerable to stress and implicated in pain perception and Fibromyalgia [26].Additionally, in a recent functional magnetic resonance imaging study, results indicate that anxiety's alteration of the network that is associated with pain modulation (including the hippocampus)underlies the manifestation of somatization [27].Consequently, in our study, the higher activation response of the hippocampus for higher hypermobility scores confirms a differential neural processing of emotional cues in hypermobility that can underlie the manifestation of anxiety and somatic symptoms through this collagen condition.

Additionally, the whole brain analysis showed that hypermobility scores were positively associated with an increased BOLD signal in some other key affective processing areas, such as the fusiform gyrus, the parahipocampal region, the middle and anterior cingulate cortex, the orbitofrontal cortex and the cerebellum. Although these results are uncorrected, it is important to highlight that all of them are key emotional processing areas. In this sense, the fusiform gyrus has been found to be critical in face processing, and the parahipocampal region in direct attention to emotional information [28, 29]. Neuroimaging studies have also described the relevance of the middle and anterior cingulate cortex in avoidance and fear learning, emotion and apathy [15], and the contribution of the orbitofrontal cortex in emotion regulation and reward in decision making [30].The cerebellum has been involved in regulating fear and pleasure responses[31].

Interestingly, the areas we have found to be associated with hypermobility scores (notably the cingulate cortex and fusiform gyrus) are crucial in the emotional brain response of subjects suffering from pathological anxiety [15]. Our suggestion is that this association may be an expression of the overrepresentation of anxiety disorders in hypermobility. Accordingly, in our sample trait anxiety was indeed strongly inter-correlated with state 
anxiety and joint hypermobility. These results are consistent with reports from previous studies. High trait anxiety has repeatedly been associated with increased responsiveness to stressors and with higher vulnerability to state anxiety and/or anxiety disorders [32, 33]. Likewise, a previous study conducted in a non-clinical population revealed the association between hypermobility and trait anxiety [7]. Our results replicate the prior ones and highlight the anxious vulnerability that high trait anxious participants may have in developing anxiety as well as in suffering from this collagen condition.

Limitations to our study include the larger number of women participants compared to men participants. However, the sample was proven to be homogeneous and the analyses were controlled for the sex variable. Another possible limitation is the use of a general population sample free from psychological or neurological disorders. This allowed us to study the emotional processing associated with hypermobility in a non-clinical sample. However, due to the high association between hypermobility and anxiety disorders, a nonclinical sample of the general population may hinder the identification of the neural underpinnings of the association

Further studies should explore the neural underpinnings of JHS and its links to the expression of anxiety disorders. Finally, as for the revealed results regarding hippocampus, future studies should explore whether hypermobile individuals present to some extent any form of attention and memory biases.

To conclude, we present a functional neuroimaging study focusing on hypermobility as a critical variable for emotional processing. Results show that hypermobility scores are associated with higher brain response in some emotion involved brain areas described to be linked to emotion regulation, anxiety and somatic symptoms. These findings increase our understanding of the mechanisms through which vulnerability to anxiety disorders and 
somatic symptoms arises in people bearing a heritable variant of collagen. A better understanding of the underpinning mechanisms of these disorders will enable improvements in their therapeutic approaches.

Competing Interest Statement: The authors have no competing interests to report

Declaration of Interest: The report represents original work that is not under consideration for publication elsewhere. The manuscript was prepared according to the journal guidelines. All authors meet criteria for authorship, disclosing all potential conflicts of interest (none), and releasing the copyright, should the manuscript be accepted for publication.

Acknowledgements: We would like to thank Ester Rosalia Martínez, Lorena Gonzalez, Marisol Picado, Laura Nuñez and Clara Canals for their collaboration in the project.

\section{REFERENCES}

[1] Beighton PH, Grahame R, Bird HA. Hypermobility of joints. In: SpringerVerlag, editor. 2ª ed. Berlín; 1989.

[2]Ross J, Grahame R. Easily missed? Joint hypermobility syndrome. Br Med J2011;342:C7167.

[3] Bulbena A, Duro JC, Mateo A, Porta M, Vallejo J. Joint hypermobility syndrome and anxiety disorders. Lancet 1988; 17;2(8612):694.

[4] Bianchi S, De Lima F, Udina M, Martin-Santos R, Crippa JA. Anxiety and joint hypermobility association: a systematic review. Rev Bras Psiquiatr 2012;34(Supl1):S53S68. 
[5]Grahame R. Hypermobility: an important but often neglected area withinrheumatology. Nat Clin Pract Rheumatol 2008; 4: 522-4.

[6]Smith TO, Easton V, Bacon H, Jerman E, Armon K, Poland F \& Macgregor AJ. The relationship between benign joint hypermobility syndrome and psychological distress: a systematic review and meta-analyses. Rheumatology 2013;274-295. doi:10.1093/rheumatology/ket317

[7] Bulbena A, Agulló A, Pailhez G, Martín-Santos R, Porta M, Guitart J, Gago J. Is joint hypermobility related to anxiety in a nonclinical population also? Psychosomatics 2004; 45(5):432-7.

[8] Bulbena A, Gago J, Pailhez G, Sperry L, Fullana MA \& Vilarroya O. Joint hypermobility syndrome is a risk factor trait for anxiety disorders: a 15-year follow-up cohort study. Gen HospPsychiatry2011; 33(4):363-70.

[9] Eccles JA, Beacher FDC, Gray MA, Jones CL, Minati L, Harrison NA \& Critchley HD. Brain structure and joint hypermobility: relevance to the expression of psychiatric symptoms. Br J Psychiatry 2012; 200:508-509.

[10]Mallorquí-Bagué N, Garfinkel SN, Engels M, Eccles JA, Pailhez G, Bulbena A, Critchley HD. Neuroimaging and psychophysiological investigation of the link between anxiety, enhanced affective reactivity and interoception in people with joint hypermobility. Front Psychol. 2014; 14;5:1162. doi: 10.3389/fpsyg.2014.01162.

[11]Blair RJ. Facial expressions, their communicatory functions and neuro-cognitive substrates. The Royal Society (Phil. Trans. R. Soc. Lond.B) 2003; 5th of February (online).

[12] Brooks SJ, Savov V, Allzén E, Benedict C, Fredriksson R, Schiöth HB. Exposure to subliminal arousing stimuli induces robust activation in the amygdala, hippocampus, 
anterior cingulate, insular cortex and primary visual cortex: A systematic meta-analysis of fMRI studies. NeuroImage2012; 59: 2962-2973.

http://dx.doi.org/10.1016/j.neuroimage.2011.09.077

[13] Smith APR, Stephan KE, Rugg MD, Dolan RJ. Task and content modulate amygdala-hippocampal connectivity in emotional retrieval. Neuron2006; 49 (4): 631-638.

[14] Stein JL, Wiedholz LM, Bassett DS, Weinberger DR, Zink CF, Mattay VS, MeyerLindenberg A. A validated network of effective amygdala connectivity. Neuro Image 2007; 36: 736-745.

[15] Holzschneider K, Mulert C. Neuroimaging in anxiety disorders. Dialogues Clin Neurosci 2011; 13(4): 453-461.

[16]Abraham A, Kaufmann C, Redlich R, Hermann A, Stark R, Stevens S, Hermann C. Self-referential and anxiety-relevant information processing in subclinical social anxiety: an fMRI study. Brain Imaging Behav 2012; Jul 8.

[17] Sheehan DV, Lecrubier Y, Harnett-Sheehan K, Janavs J, Weiller E, Bonora LI, Keskiner A, Schinka J, Knapp E, Sheehan MF, Dunbar GC. "The Mini-International Neuropsychiatric Interview (M.I.N.I.): the development and validation of a structured diagnostic psychiatric interview for DSM-IV and ICD-10". J Clin Psychiatry 1998; 59 Suppl 20: 22-33. In its Spanish 5.0.0 version validated in our country by Fernando L, Bobes J, Gibert J, Soto M, Soto O. Instituto IAP. 1995.

[18] First MB, Spitzer RL, Gibbon M, Williams JBW. Entrevista Clínica Estructurada para los Trastornos del Eje I del DSM-IV. Versión Clínica (SCID-I). Barcelona, Masson, S.A;1999. 
[19] Spielberger CD, Gorusch RL \&Lushene. Cuestionario de Ansiedad Estado-Rasgo. Ed: TEA, Madrid;1986.

[20] Beighton PH. Hypermobility scoring. Br J Rheumatol 1988;27:163.

[21] Gur RC, Sara R, Hagendoorn M, Marom O, Hughett P, Macy L, Turner T, Bajcsy R, Posner A \& Gur RE. A method for obtaining 3-dimensional facial expressions and its standardization for use in neurocognitive studies. J. Neurosci. Methods; 2002 115, 137-43.

[22] Maldjian, JA, Laurienti, PJ, Burdette, JB, Kraft RA. An Automated Method for Neuroanatomic and Cytoarchitectonic Atlas-based Interrogation of fMRI Data Sets.

NeuroImage 2003; 19:1233-1239.

[23]Maldjian JA, Laurienti PJ, Burdette JH. Precentral Gyrus Discrepancy in Electronic Versions of the Talairach Atlas. Neuroimage 2004; 21(1) 450-455.

[24]Smith DM, Mizumori SJ. Hippocampal place cells, context, and episodic memory. Hippocampus2006;16(9):716-29.7

[25]Pantazatos SP, Talati A, Schneier FR, Hirsch J. Reduced anterior temporal and hippocampal functional connectivity during face processing discriminates individuals with social anxiety disorder from healthy controls and panic disorder, and increases following treatment. Neuropsychopharmacology 2014;39(2):425-34. doi: 10.1038/npp.2013.211

[26] Aoki Y, Inokuchi R, Suwa H. Reduced N-acetylaspartate in the hippocampus in patients with fibromyalgia: a meta-analysis. Psychiatry Res 2013; 30;213(3):242-8. doi: 10.1016/j.pscychresns.2013.03.008. 
[27] Gondo M, Moriguchi Y, Kodama N, Sato N, Sudo N, Kubo C, Komaki G. Daily physical complaints and hippocampal function: an fMRI study of pain modulation by anxiety. Neuroimage 2012; 15;63(3):1011-9. doi: 10.1016/j.neuroimage.2012.07.025.

[28] Ziaei M, Peira N, Persson J. Brain systems underlying attentional control and emotional distraction during working memory encoding. Neuroimage 2013.doi: 10.1016/j.neuroimage.2013.10.048.

[29]Pujol J, Harrison BJ, Ortiz H, Deus J, Soriano-Mas C, López-Solà M, Yücel M, Perich $\mathrm{X}$, Cardoner N. Influence of the fusiform gyrus on amygdala response to emotional faces in the non-clinical range of social anxiety. PsycholMed 2009; 39(7):1177-87.

[30]Golkar A, Lonsdorf TB, Olsson A, Lindstrom KM, Berrebi J, Fransson P, Schalling M, Ingvar M, Öhman A. Distinct contributions of the dorsolateral prefrontal and orbitofrontal cortex during emotion regulation. PLoS One2012; 7(11):e48107. doi: 10.1371/journal.pone.0048107.

[31] Wolf U, Rapoport MJ, Schweizer TA. Evaluating the affective component of the cerebellar cognitive affective syndrome. J. Neuropsychiatry Clin. Neurosci 2009; 21 (3): 245-53.

[32] Endler NS, Kocovski NL. State and traït anxiety revisited. J Anxiety Disord 2001;15(3):231-45.

[33] Wilken JA, Smith BD, Tola K, Mann M. Trait anxiety and prior exposure to nonstressful stimuli: effects on psychophysiological arousal and anxiety. Int J Psychophysiol 2000; 37(3):233-42. 
Table 1. Self-reported anxiety clinical measures and hypermobility scores $(n=51)$

\begin{tabular}{lccccc}
\hline & Min-Max & Mean & $\begin{array}{c}\text { Standard } \\
\text { deviation }\end{array}$ & \multicolumn{2}{c}{$r$ - Pearson correlation index ( $p$ value $)$} \\
\cline { 5 - 6 } & & & & State anxiety & Trait anxiety \\
\hline Age & $21-45$ & 33.31 & 4.93 & --- & --- \\
\hline State anxiety * & $3-35$ & 14.43 & 7.24 & --- & $0.507(<0.001)$ \\
\hline Trait anxiety * & $2-33$ & 16.41 & 7.03 & $0.507(<0.001)$ & --- \\
\hline Hypermobility ** & $0-9$ & 2.94 & 2.64 & $0.044(0.758)$ & $0.303(0.031)$
\end{tabular}

*Measured by means of STAI state-trait anxiety inventory.**Beighton assessment, maximum score=9.

Table 2: Whole-brain analysis of the activity seen in crying face versus neutral face images with correlation analysis with hypermobility scores. $(\mathrm{p}=0.001$, uncorrected).

\begin{tabular}{lcccccc}
\hline Brain Area & Side & Cluster Size & Coordinates & \multicolumn{1}{c}{-Value } \\
\hline Middle Cingulate cortex & $\mathrm{L}$ & 240 & -16 & -30 & 46 & 4.90 \\
\hline Middle Cingulate cortex & $\mathrm{R}$ & 80 & 14 & -18 & 48 & 3.47 \\
\hline Anterior Cingulate cortex & $\mathrm{R}$ & 117 & 16 & 36 & 24 & 3.77 \\
\hline Anterior Cingulate cortex & $\mathrm{R}$ & 14 & 14 & 50 & 18 & 3.48 \\
\hline Hippocampus & $\mathrm{L}$ & 79 & -40 & -22 & -18 & 4.19 \\
\hline Parahippocampalgyrus & $\mathrm{L}$ & 62 & -20 & -30 & -16 & 3.98 \\
\hline Fusiform gyrus & $\mathrm{L}$ & 62 & -26 & -36 & -16 & 3.75 \\
\hline Fusiform gyrus & $\mathrm{R}$ & 16 & 30 & -4 & -38 & 3.72 \\
\hline Fusiform gyrus & $\mathrm{R}$ & 16 & 36 & -10 & -38 & 3.39 \\
\hline Supplementary motor area & $\mathrm{R}$ & 80 & 6 & -16 & 50 & 3.41 \\
\hline Medial Orbital Frontal & $\mathrm{R}$ & 57 & 14 & 62 & -4 & 3.81 \\
\hline
\end{tabular}




\begin{tabular}{lllllll}
\hline Sub Orbital Frontal & $\mathrm{R}$ & 57 & 16 & 54 & -8 & 3.56 \\
\hline Postcentral gyrus & $\mathrm{L}$ & 33 & -40 & -22 & 54 & 3.74 \\
\hline Cerebelum_4_5 & $\mathrm{R}$ & 26 & 28 & -36 & -32 & 3.62 \\
\hline
\end{tabular}
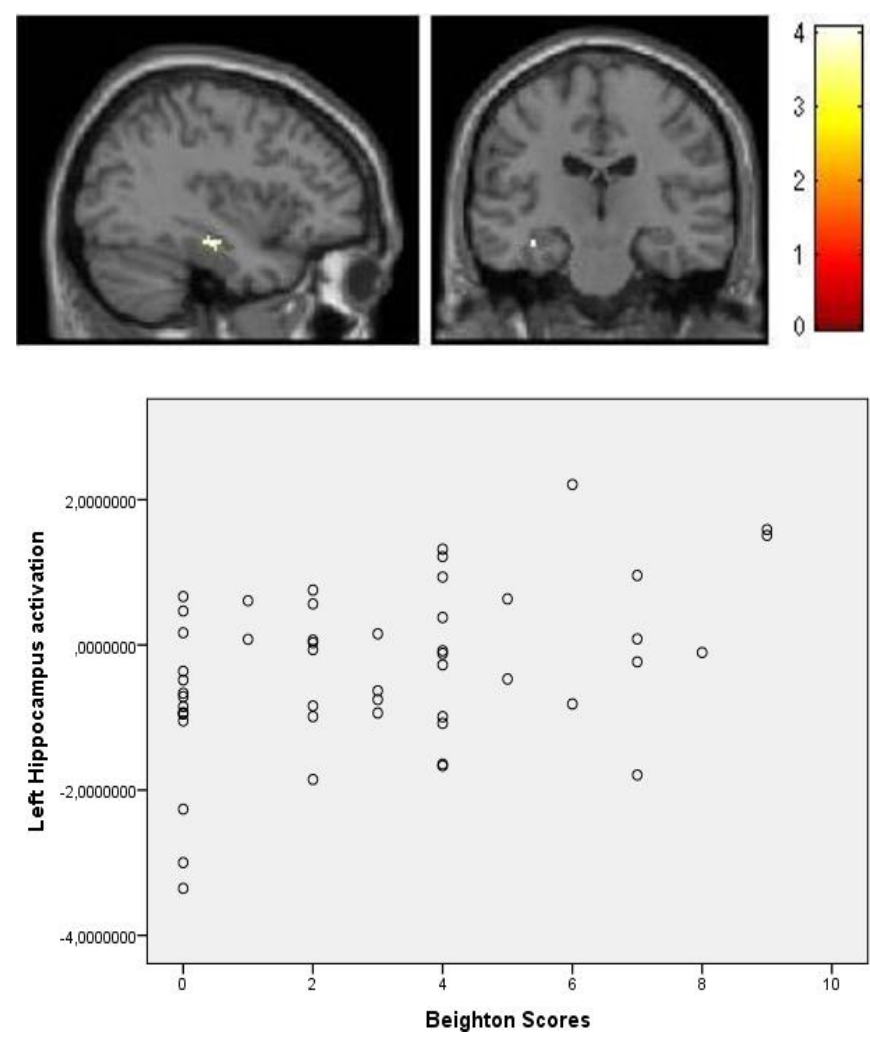

Figure 1.Activations obtained from a t-test over the linear regression between the cry face $>$ neutral face contrast and Beighton hypermobility scores within the Hippocampus Left ROI ( $<<0.05$ FWE-Corrected). . At the top of the figure, the brain mapping images show the activated clusters. At the lower part of the figure, the mean contrast value for each individual in the area of significant group correlation is plotted against the corresponding Beighton Hypermobility score. 